\title{
Comparison between the numerical solutions and the Thomas-Fermi approximation for atomic-molecular Bose-Einstein condensates
}

\author{
L. S. F. Santo:* \\ Departamento de Ciências Exatas e da Terra, \\ Universidade Federal de São Paulo, Campus Diadema, \\ Rua Prof. Artur Riedel, 275, Jd. Eldorado, 09972-270 Diadema, SP, Brazil \\ M. O. C. Pirest \\ Centro de Ciências Naturais e Humanas, Universidade Federal do ABC, \\ Rua Santa Adélia 166, 09210-170, Santo André, SP, Brazil \\ Davi Giugnd \\ Instituto de Física, Universidade de São Paulo, \\ CP 66318, 05315-970, São Paulo-SP, Brazil
}

\begin{abstract}
We study the stationary solution of an atomic Bose-Einstein condensate coupled coherently to a molecular condensate with both repulsive and attractive interspecies interactions confined in an isotropic harmonic trap. We use the Thomas-Fermi approximation and find four kinds of analytical solution for the cases. These analytical solutions are adopted as trial function for the diffusive numerical solution of the Gross-Pitaevskii equations. For the repulsive interspecies interaction, the case in which the atomic and molecular wavefunctions are out-phase, the densities have similar profiles for both methods, however, the case where the wavefunctions are in-phase, there are considerable difference between the density profiles. For the attractive interspecies interaction, there are two cases in the Thomas Fermi approximation where the wavefunctions are in-phase. One of them has numerical solution that agree with the approximation and the other does not have corresponding numerical solution.
\end{abstract}

PACS numbers: 05.30.Jp,03.75.Hh,03.65.Ge,33.80.Ps,03.75.Mn

\footnotetext{
*Electronic address: leosioufi@gmail.com

${ }^{\dagger}$ Electronic address: marcelo.pires@ufabc.edu.br

${ }^{\ddagger}$ Electronic address: dgiugno@if.usp.br
} 


\section{INTRODUCTION}

The experimental observation of the Bose-Einstein condensate (BEC) from ultra-cold and dilute alkali gases allowed a lot of important applications for, and investigations on, cold atomic gases [1 3]. Many researchers have intended to produce a mixture of atomic and molecular BEC (AMBEC) with atom-to-molecule conversion by the Raman photo-association process [4] or by the Feshbach resonance technique [5]. At present, it is possible to convert fermionic and bosonic atoms into homonuclear [6] and heteronuclear [7, 8] diatomic molecules using the Feshbach resonance technique. Moreover, there is some experimental support to the coherent binding of bosonic atoms into molecules through a Raman photo-association process [4]. Among these possibilities, only the molecular BEC composed of fermionic atoms have been observed, i. e., the AMBEC has not yet been detected in laboratory at the present moment.

One of the main reasons for the study of the AMBEC is the perspective of understanding molecular formation and destruction stimulated by a coherent coupling from macroscopic quantum states 9]. Then, a feature of the AMBEC is the relative quantum phase between atomic and molecular wave-functions, which offers an atom-optic analogue to the second-harmonic generation in non-linear optics [10]. The quantum relative phase appears in any mixture of coherent state BEC with conversion between particles [11, 12]. Moreover, for the references [13, 14], another characteristic is that the AMBEC presents the phase separation, where the AMBEC does not exist as a mixture in equilibrium, but rather producing pure molecular BEC and AMBEC domain structures.

In the mean-field model, the dynamics of the AMBEC is described by two coupled GrossPitaevskii equations (GPE) for the macroscopic wave function of the atomic and molecular species. Many authors use the Thomas-Fermi approximation (TFA) for solving the GPE [10, 13] in the limit where the number of particles is large. In this approximation, the kinetic energy terms in the GPE are neglected. The main advantage of this procedure is the possibility of obtaining analytical solutions for the GPE.

In previous works, the validity of the TFA in the AMBEC was investigated from different points of view. In work [10], the authors analysed an AMBEC coupled via photo-association process and excluded the TFA for the self-trapped states where the atom-molecule interaction is attractive and the relative phase is $\pi$. In reference [13], the authors adopted the TFA in the GPE for the AMBEC coupled via the Feshbach resonance. The authors concluded that the TFA required a slow spatial variation of the AMBEC to be valid, which fails to occur at the boundary of the two phases. Then, 
the previous works agree that there are problems in the spatial distributions when one adopts the TFA. However, the reference [13] did not compare the numerical solution of the GPE for the AMBEC to the TFA solutions at the same conditions.

In this work, we have compared the TFA to the numerical solution of the GPE for the AMBEC in the available conditions. This paper have been organized as follows. In section I, we have presented the model and we have derived the GPE. The TFA is shown in the section III. The numerical implementation and the discussion have been dealt with in sections [V] and $\mathrm{V}$, respectively. The conclusion and remarks have been left for section VI.

\section{THE MODEL AND THE GROSS-PITAEVSKII EQUATIONS}

We have considered that the AMBEC is within a spherical harmonic trap with frequency $\omega$ for both species, where the atom-to-molecule conversion can be either via Feshbach resonance or via Raman photo-association. Moreover, we have accounted for the molecule mass as being twice the atomic mass, $m$. In the mean-field theory, the total energy in units of $\hbar \omega$ is given by:

$$
\begin{aligned}
\langle H\rangle & =\int\left(-\frac{\zeta}{2} \phi_{1}^{*}(r) \nabla^{2} \phi_{1}(r)+\frac{1}{2} r^{2}\left|\phi_{1}(r)\right|^{2}+\frac{U_{1}}{2}\left|\phi_{1}(r)\right|^{4}\right) d^{3} r+ \\
& +\int\left(-\frac{\zeta}{4} \phi_{2}^{*}(r) \nabla^{2} \phi_{2}(r)+r^{2}\left|\phi_{2}(r)\right|^{2}+\frac{U_{2}}{2}\left|\phi_{2}(r)\right|^{4}\right) d^{3} r+ \\
& +\int\left(U_{12}\left|\phi_{1}(r)\right|^{2}\left|\phi_{2}(r)\right|^{2}+\frac{\alpha}{2}\left(\phi_{1}^{2}(r) \phi_{2}^{*}(r)+\phi_{1}^{2 *}(r) \phi_{2}(r)\right)\right) d^{3} r .
\end{aligned}
$$

Here $\phi_{1}(r)$ and $\phi_{2}(r)$ are the atomic and molecular wave functions in units of $(\hbar / m \omega)^{-3 / 4}$. The spatial radial coordinate $r$ is in units of $(m \omega / \hbar)^{1 / 2}$. The parameter $\zeta$ can be either 0 or 1 depending on whether the TFA is used or not. The parameters $U_{1}, U_{2}$ and $U_{12}$ are the atomic, molecular and atomic-molecular interaction strengths, respectively. We have considered that $U_{1}$ and $U_{2}$ are both positive and $U_{12}$ can be either positive or negative. We have defined the parametric coupling strength $\alpha$ as a positive parameter because the real term $\left(\phi_{1}^{2}(r) \phi_{2}^{*}(r)+\phi_{1}^{2 *}(r) \phi_{2}(r)\right)$ can be either positive or negative. The interaction strengths and the parametric coupling strength are measured respectively in units of $(\hbar \omega)^{-1}(\hbar / m \omega)^{-3 / 2}$ and $(\hbar \omega)^{-1}(\hbar / m \omega)^{-3 / 4}$. We have neglected the energy for creating a molecule from two atoms.

We have obtained the wave functions $\phi_{1}(r)$ and $\phi_{2}(r)$ from the variational method. We have used the linear functional $\langle H\rangle-\mu\langle N\rangle$, where the chemical potential, $\mu$, is the Lagrange's multiplier and $\langle N\rangle=\left\langle N_{1}\right\rangle+2\left\langle N_{2}\right\rangle=\int\left(\left|\phi_{1}(r)\right|^{2}+2\left|\phi_{2}(r)\right|^{2}\right) d^{3} r$ is the average of the total number of particles. 
The variational method provides the following GPE,

$$
\begin{gathered}
-\left(\frac{\zeta}{2 r^{2}}\right) \frac{\partial}{\partial r}\left(r^{2} \frac{\partial \phi_{1}(r)}{\partial r}\right)+\left(-\widetilde{\mu}(r)+U_{1}\left|\phi_{1}(r)\right|^{2}+U_{12}\left|\phi_{2}(r)\right|^{2}\right) \phi_{1}(r)+\alpha \phi_{1}^{*}(r) \phi_{2}(r)=0 \\
-\left(\frac{\zeta}{4 r^{2}}\right) \frac{\partial}{\partial r}\left(r^{2} \frac{\partial \phi_{2}(r)}{\partial r}\right)+\left(-2 \widetilde{\mu}(r)+U_{2}\left|\phi_{2}(r)\right|^{2}+U_{12}\left|\phi_{1}(r)\right|^{2}\right) \phi_{2}(r)+\frac{\alpha}{2} \phi_{1}^{2}(r)=0
\end{gathered}
$$

where $\widetilde{\mu}(r)=\mu-\frac{r^{2}}{2}$ is the effective local chemical potential in units of $\hbar \omega$.

We have replaced $\phi_{1}(r)=\left|\phi_{1}(r)\right| e^{i \theta_{1}}$ and $\phi_{2}(r)=\left|\phi_{2}(r)\right| e^{i \theta_{1}} \beta$ in the equations (21) and (31), where $\beta=e^{i \theta}$ is a function of the relative phase, $\theta$, between $\phi_{1}(r)$ and $\phi_{2}(r)$. Then the two equations read:

$$
\begin{aligned}
& -\left(\frac{\zeta}{2 r^{2}}\right) \frac{\partial}{\partial r}\left(r^{2} \frac{\partial\left|\phi_{1}(r)\right|}{\partial r}\right)+\left(-\widetilde{\mu}(r)+U_{1}\left|\phi_{1}(r)\right|^{2}+U_{12}\left|\phi_{2}(r)\right|^{2}\right)\left|\phi_{1}(r)\right|+\alpha \beta\left|\phi_{1}(r)\right|\left|\phi_{2}(r)\right|=0, \\
& -\left(\frac{\zeta}{4 r^{2}}\right) \frac{\partial}{\partial r}\left(r^{2} \frac{\partial\left|\phi_{2}(r)\right|}{\partial r}\right)+\left(-2 \widetilde{\mu}(r)+U_{2}\left|\phi_{2}(r)\right|^{2}+U_{12}\left|\phi_{1}(r)\right|^{2}\right)\left|\phi_{2}(r)\right|+\frac{\alpha}{2} \beta\left|\phi_{1}(r)\right|^{2}=0 .
\end{aligned}
$$

The equations (4) and (5) can be solved only for $\beta= \pm 1$. We have classified the solutions $\beta=1$ and $\beta=-1$ respectively as " in-phase" and "out-of-phase". The parametric coupling strength, $\alpha$ determines effectively the repulsion $(\beta=+1)$ and the attraction $(\beta=-1)$ between the atomic and molecular BEC. These results and classification have appeared in precedent work [10].

The solutions of the equations (4) and (5) are different for each $\zeta$ value. These solutions have distinct behaviours and, therefore, should be analysed separately. The analysis for each kind of solution shall be done in the next two sections.

\section{THOMAS-FERMI APPROXIMATION}

Considering $\zeta=0$ in the equations (44) and (5), we have found three kinds of solutions at a specific position $r$. These kinds are: vacuum where $\left|\phi_{1}(r)\right|=\left|\phi_{2}(r)\right|=0(V)$, pure molecular solution where $\left|\phi_{2}(r)\right|^{2}=\frac{2 \widetilde{\mu}(r)}{U_{2}}$ and $\left|\phi_{1}(r)\right|=0(P M)$, mixed solution where $\left|\phi_{1}(r)\right| \neq 0$ and $\left|\phi_{2}(r)\right| \neq 0(A M)$. There is no pure atomic solution where $\left|\phi_{1}(r)\right| \neq 0$ and $\left|\phi_{2}(r)\right|=0$ because $\alpha \neq 0$. For each radius $r$, the equations (4) and (5) may have a different kind of solution.

The mixed solutions of (4) and (5) can be found by solving two coupled cubic equations [13]. For the sake of simplicity, we have restricted ourselves to the case where the atomic-molecular interaction strength is given by $U_{12}=\gamma \sqrt{U_{1} U_{2}}$, where $\gamma= \pm 1$. This restriction reduces the three solutions of the cubic equations to just two. The situations $\gamma=+1$ and $\gamma=-1$ correspond 
respectively to $U_{12}>0$ (atomic-molecular repulsion) and $U_{12}<0$ (atomic-molecular attraction). Taking this into consideration, the mixed solutions are given by,

$$
\begin{gathered}
\left|\phi_{1}(r)\right|^{2}=\frac{\widetilde{\mu}(r)}{U_{1}}-\frac{\alpha \beta}{U_{1}}\left|\phi_{2}(r)\right|-\gamma \sqrt{\frac{U_{2}}{U_{1}}}\left|\phi_{2}(r)\right|^{2}, \\
\left|\phi_{2}\right|=\beta \gamma B(r)+\delta \sqrt{B^{2}(r)+\gamma C(r)},
\end{gathered}
$$

where $\delta= \pm 1$ labels the two solutions of (4) and (15),

$$
B(r)=\frac{1}{3 \alpha}\left(\widetilde{\mu}(r)\left(\gamma-2 \sqrt{\frac{U_{1}}{U_{2}}}\right)-\frac{\alpha^{2}}{2 \sqrt{U_{1} U_{2}}}\right),
$$

and

$$
C(r)=\frac{\widetilde{\mu}(r)}{3 \sqrt{U_{1} U_{2}}}
$$

We have classified the mixed solutions according to the sequence of signals $A M_{\gamma \beta \delta}$. For example, the solution $A M_{-++}$has $\gamma=-1, \beta=+1$ and $\delta=+1$. In contrast with $\beta$ and $\gamma$, the parameter $\delta$ does not indicate the atomic-molecular attraction or repulsion.

We have excluded those solutions that do not have a mathematical or physical meaning. Firstly, on mathematical grounds we have excluded those solutions for which $\left|\phi_{1}(r)\right|^{2}<0,\left|\phi_{2}(r)\right|<0$ and $\left|\phi_{2}(r)\right| \notin \mathbb{R}\left(B^{2}-\gamma C<0\right)$. Next, we have rejected the solutions where the total density, $\left|\phi_{1}\right|^{2}+2\left|\phi_{2}\right|^{2}$, increases with $r$. This is because the trap forces the particles towards the center of the system.

Another physical criterion is the continuity of the density. To ensure this property, it is necessary to define new continuous functions $\left|\phi_{1}(r)\right|^{2}$ and $\left|\phi_{2}(r)\right|$, where a different $r$ corresponds to a different solution. Indeed, we have one situation where we are not able to build a continuous function for $\left|\phi_{1}(r)\right|^{2}$ and $\left|\phi_{2}(r)\right|$.

Finally, we have ignored the trivial vacuum solution for all $r$ because it does not satisfy the normalization condition $N \neq 0$.

According to these criteria, the solutions $A M_{++-}$and $A M_{---}$are excluded for all $r$. The solutions $A M_{--+}$and $A M_{+--}$are rejected by the mathematical criteria for $r<\sqrt{2 \mu}$ and by the physical criteria for $r>\sqrt{2 \mu}$.

Then, we have five possible ways to describe the atomic and molecular densities for all $r$ in the TFA when we have restricted $U_{1}>U_{2} / 4$. This restriction is close to what is observed in realistic solutions. Otherwise, all the following analysis would be pointless. In the table $\llbracket$ the classification 
TABLE I: Kinds of solution in the TFA.

\begin{tabular}{|c|c|c|c|}
\hline Classification & $A M$ & $V$ & $P M$ \\
\hline \hline$S_{M}$ & - & $r \geq \sqrt{2 \mu}$ & $r \leq \sqrt{2 \mu}$ \\
\hline$S_{+++}$ & $r \leq \sqrt{2 \mu}$ & $r \geq \sqrt{2 \mu}$ & - \\
\hline$S_{-+-}$ & $r \leq \sqrt{2 \mu}$ & $r \geq \sqrt{2 \mu}$ & - \\
\hline$S_{-++}$for $\mu>\mu_{+}$ & $r \leq \sqrt{2\left(\mu-\mu_{+}\right)}$ & $r \geq \sqrt{2 \mu}$ & $\sqrt{2\left(\mu-\mu_{+}\right)} \leq r \leq \sqrt{2 \mu}$ \\
\hline$S_{+-+}$ & $\sqrt{2\left(\mu-\mu_{-}\right)} \leq r \leq \sqrt{2\left(\mu-\mu_{d}\right)}$ & $r \geq \sqrt{2\left(\mu-\mu_{d}\right)}$ & $r \leq \sqrt{2\left(\mu-\mu_{-}\right)}$ \\
\hline
\end{tabular}

of these five possibilities is shown and the intervals of the different domain solutions are indicated. The expressions for $\mu_{ \pm}$and $\mu_{d}$ that appeared in table $\llbracket$ are defined by:

$$
\begin{gathered}
\mu_{d}=-\frac{\alpha^{2}}{\left(\sqrt{U_{2}}-2 \sqrt{U_{1}}\right)^{2}}\left(\sqrt{\frac{U_{2}}{U_{1}}}+1-\sqrt{\left.\frac{3}{4}\left(\frac{U_{2}}{U_{1}}\right)+3 \sqrt{\frac{U_{2}}{U_{1}}}\right)},\right. \\
\mu_{ \pm}=\frac{2 \alpha^{2}}{\left(\sqrt{U_{2}} \pm 2 \sqrt{U_{1}}\right)^{2}} .
\end{gathered}
$$

The wave functions $\phi_{1}(r)$ and $\phi_{2}(r)$, in the cases $S_{M}, S_{+++}, S_{-+-}$and $S_{-++}$, are continuous. However the wave functions are discontinuous for the case $S_{+-+}$at $r=\sqrt{2\left(\mu-\mu_{d}\right)}$. These discontinuities have already appeared in previous works [13].

We have not represented the solution $S_{M}$ because this case is similar to the single component BEC. For the other four possibilities, we have compared the TFA to the numerical solution, and this is dealt with in the next section.

\section{NUMERICAL SOLUTION}

We have solved the equations (44) and (5) for $\zeta=1$ by applying the relaxation algorithm [1518] for the GPE. The algorithm consists of a method that provides a numerical solution of the GPE by considering an imaginary time variable, $\tau$. Redefining the parametrized GPE by the imaginary time, we can obtain two coupled non-linear diffusion equations. The propagation of the trial functions, using this diffusion equation, provides the numerical stationary solution at large imaginary times. In order to compare the numerical solutions to the TFA solutions, we have used the Thomas-Fermi profiles as the trial functions.

For the implementation of the relaxation algorithm, we have first rewritten the GPE (44) and 
(5) considering $U_{12}=\gamma \sqrt{U_{1} U_{2}}$, as

$$
\left\{\begin{array}{l}
\frac{d^{2} \psi_{1}(r)}{d r^{2}}=-2 \widetilde{\mu}(r) \psi_{1}(r)+2 U_{1}\left(\frac{\psi_{1}^{3}(r)}{r^{2}}\right)+2 \gamma \sqrt{U_{1} U_{2}}\left(\frac{\psi_{2}^{2}(r) \psi_{1}(r)}{r^{2}}\right)+2 \beta \alpha\left(\frac{\psi_{2}(r) \psi_{1}(r)}{r}\right) \\
\frac{d^{2} \psi_{2}(r)}{d r^{2}}=-8 \widetilde{\mu}(r) \psi_{2}(r)+4 U_{2}\left(\frac{\psi_{2}^{3}(r)}{r^{2}}\right)+4 \gamma \sqrt{U_{1} U_{2}}\left(\frac{\psi_{1}^{2}(r) \psi_{2}(r)}{r^{2}}\right)+2 \beta \alpha\left(\frac{\psi_{1}^{2}(r)}{r}\right)
\end{array},\right.
$$

where the functions $\psi_{1}(r)$ and $\psi_{2}(r)$ are related to the atomic and molecular wave functions by $\left|\phi_{1}(r)\right|=\psi_{1}(r) / r$ and $\left|\phi_{1}(r)\right|=\psi_{1}(r) / r$.

We have introduced the imaginary time variable in the equations (6). This imaginary time is able to lead the trial function to the stationary solution by a diffusion process. In fact, this process must be possible if we consider the set of the non-linear diffusion equations,

$$
\left\{\begin{array}{l}
\frac{\partial \psi_{1}(r)}{\partial \tau}=\frac{\partial^{2} \psi_{1}(r)}{\partial r^{2}}+2 \widetilde{\mu}(r) \psi_{1}(r)-2 U_{1}\left(\frac{\psi_{1}^{3}(r)}{r^{2}}\right)-2 \gamma \sqrt{U_{1} U_{2}}\left(\frac{\psi_{2}^{2}(r) \psi_{1}(r)}{r^{2}}\right)-2 \beta \alpha\left(\frac{\psi_{2}(r) \psi_{1}(r)}{r}\right) \\
\frac{\partial \psi_{2}(r)}{\partial \tau}=\frac{\partial^{2} \psi_{2}(r)}{\partial r^{2}}+8 \widetilde{\mu}(r) \psi_{2}(r)-4 U_{2}\left(\frac{\psi_{2}^{3}(r)}{r^{2}}\right)-4 \gamma \sqrt{U_{1} U_{2}}\left(\frac{\psi_{1}^{2}(r) \psi_{2}(r)}{r^{2}}\right)-2 \beta \alpha\left(\frac{\psi_{1}^{2}(r)}{r}\right)
\end{array}\right.
$$

These equations provide a convergent diffusion process and several solutions coexist within numerical precision for a large imaginary time $\tau \rightarrow \infty$. At this limit, the trial solutions propagate to the solution of equations (4) and (5).

For these new functions $\psi_{1}$ and $\psi_{2}$, the normalization conditions are given by,

$$
\left\{\begin{array}{l}
\int_{0}^{\infty} \psi_{1}^{2}(r) d r=\frac{N_{1}}{4 \pi} \\
\int_{0}^{\infty} \psi_{2}^{2}(r) d r=\frac{N_{2}}{4 \pi}
\end{array},\right.
$$

with the requirement that the wave functions must vanish far from the trap center. In the same way, the non-linear term inside Eq. (6) must eventually become negligible compared to the other two terms. The asymptotic form has the behaviour $\psi_{1}(r) \approx c_{1} e^{-r^{2} / 4+(\mu-1 / 2) \ln (r)}$ and $\psi_{2}(r) \approx$

$c_{2} e^{-r^{2} / 4+(4 \mu-1 / 2) \ln (r)}$, where $c_{1}=\sqrt{N_{1} / \Gamma(\mu)}$ and $c_{2}=\sqrt{N_{2} /(2 \Gamma(4 \mu))}$ were determined by the wave function normalization, with $\Gamma(x)$ being the gamma function.

For the limit $r \rightarrow 0$, the non-linear term inside Eq. (6) approaches a constant due to the regularity of the wave function at $r=0$. Then we can write $\psi_{1}(r) \approx \psi_{1}^{\prime}(0) r$ and $\psi_{2}(r) \approx \psi_{2}^{\prime}(0) r$ in this limit.

We have kept the total particle number fixed in the whole diffusion process. However, the chemical potential is changed at every iteration step. The convergence of the chemical potential has been used as a criterion to stop the diffusion process.

\section{DISCUSSION}

We have compared the TFA to the numerical solution for each spatial distribution. This correspondence of the TFA to the numerical solution is given by the input of the diffusive numerical 
procedure.

The numbers of particles in our simulation are compatible with the usual numbers of particles of experimental atomic BEC's, namely, $10^{4}, 2 \times 10^{4}, 5 \times 10^{4}, 10^{5}, 2 \times 10^{5}, 5 \times 10^{5}$ and $10^{6}$. We have adjusted the chemical potential in order to obtain these numbers of particles.

For the repulsive atom-molecule interaction $\left(U_{12}>0\right)$ corresponding to $\gamma=+1$ cases, we have employed the parameters used in theoretical work [13] which is based on values in [9], $U_{1}=0.1$, $U_{2}=0.036$ and $\alpha=1.08$. For the attractive atom-molecule interaction $\left(U_{12}<0\right)$ corresponding to $\gamma=-1$, we have used those from [4, 10] instead, namely $U_{1}=0.062, U_{2}=0.12$ and $\alpha=1.09$. As for the Cusack data [10], the only difference between our set of parameters and theirs is the value of $U_{12}$. We have been set to $U_{12}=-\sqrt{U_{1} U_{2}} \approx-0.0876$ but the reference [10] had been fixed exactly at $U_{12}=-0.087$. Even so, our value for $U_{12}$ is compatible with the experimental measurement $U_{12}=-0.087 \pm 0.07$ [4, 10]. All sets of parameters are inspired on the diatomic molecules created at rest in a dilute Bose-Einstein condensate of rubidium- 87 atoms with coherent free-bound stimulated Raman transitions [4, 9].

For these parameters, the behaviour of the chemical potential and the central density as function of number of particles can be seen in figures 1 and 2, In the figure 1, we have graphed the chemical potential in the TFA and the modular variation of the chemical potential obtained by the TFA solution and by the diffusive numerical solution. In the TFA, the chemical potential of the $S_{+++}$ and $S_{-+-}$cases is larger than $S_{-++}$and $S_{+-+}$cases for any numbers of particles. Analysing the figure 1b), the $S_{+++}$case present the modular variation more significant than $S_{-++}$and $S_{+-+}$ cases. Notice that for the $S_{-+-}$we have no numerical solutions at all. Hence we shall not display any relative variation curves.

Furthermore, in the fig. 2, we have produced the graphs for the atomic and molecular central density as function of the number of particles in the TFA. In graphs $2 \mathrm{a}$ ) and $2 \mathrm{~b}$ ), four cases are considered. In the graphs $2 \mathrm{r}$ ) and $2 \mathrm{~d}$ ), we have plotted the modular variation of the atomic and molecular central density. In both, the modular variation of the central density in the $S_{+++}$case is larger than the others.

For the $S_{+++}$case, there is not available numerical solution for $N>7 \times 10^{5}(\mu>47$.). In this case, there is a considerable difference in the central densities of the profiles provided by the numerical calculation and the TFA (see in the fig. 2 and 3). The diffusion process in the numerical solution concentrate the densities in the center of the trap. Moreover, in the TFA, the molecular 

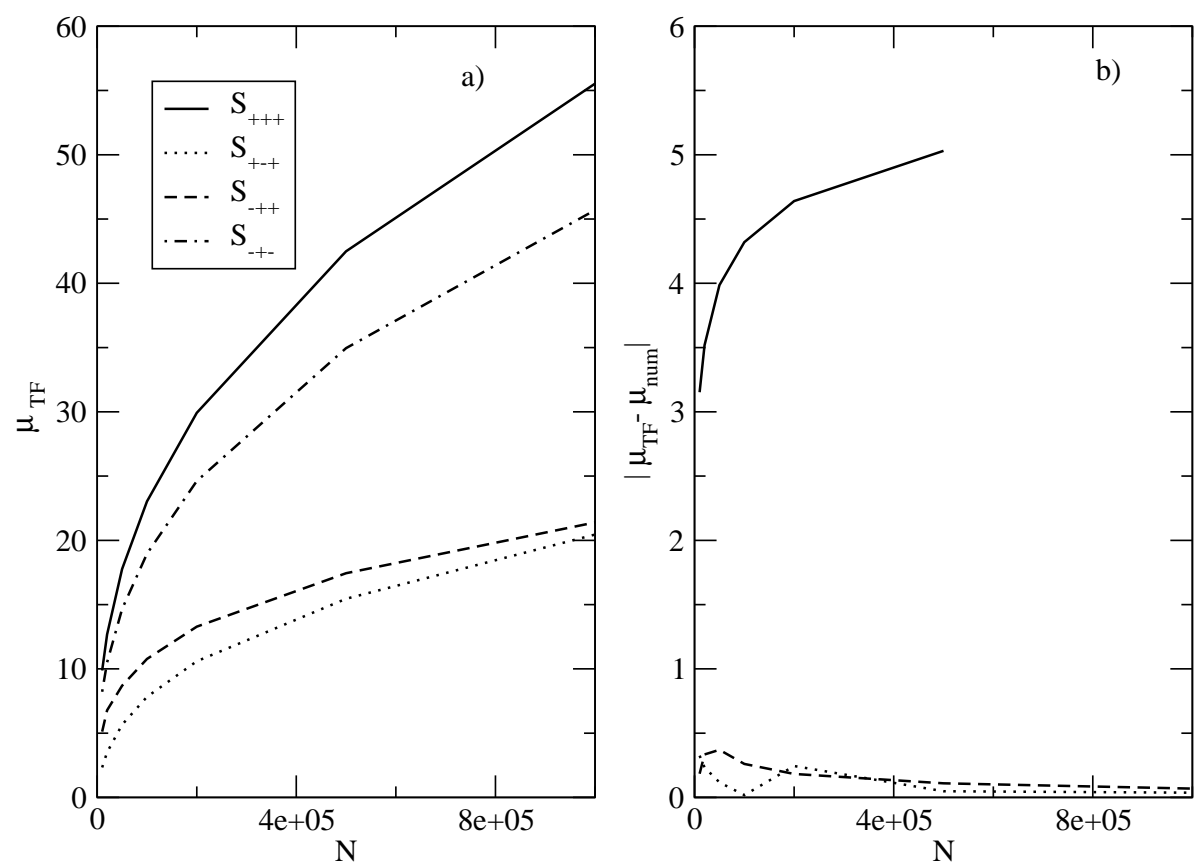

FIG. 1: The chemical potential in the TFA (fig. a)) and its variation between the TFA and numerical solution (fig. b)), $\left|\mu_{T F}-\mu_{\text {num }}\right|$ as functions of the number of particles in four cases, $S_{+++}$(solid line), $S_{+-+}$(dotted line), $S_{-++}$(dashed line) and $S_{-+-}$(dotted-dashed line). There is no variation for the $S_{-+-}$ case because we do not have the numerical solution for this case.

and atomic densities are found to be continuous, but their first spatial derivatives present a firstorder discontinuity when $r=\sqrt{2 \mu}$. On the other hand, we have found that the densities and the first derivatives are continuous everywhere in the numerical solution.

For the $S_{+-+}$case (see in the fig. (4) we have found a good agreement between the TFA and the numerical solution. Although the agreement, for the TFA there are discontinuities not only in the first spatial derivative of the spatial distributions but also in the distributions themselves at the point $r_{d}=\sqrt{2\left(\mu-\mu_{d}\right)}$ and, for $r>r_{d}$, there is vacuum. Moreover, in the case where $\mu>\mu_{-}$ (for our parameters, $\mu>10.90$ ), the $S_{+-+}$case at the TFA presents a discontinuity in the first spatial derivatives of both the atomic and molecular densities, at the point $r_{-}=\sqrt{2\left(\mu-\mu_{-}\right)}$. For $r<r_{-}$, the spatial distribution becomes purely molecular. While in the numerical solution, there is no phase separation between the atomic and molecular distribution, and no discontinuities of any kind have been seen. However, in the region of purely molecular density in the TFA, the atomic density provided by the numerical solution is almost null. 

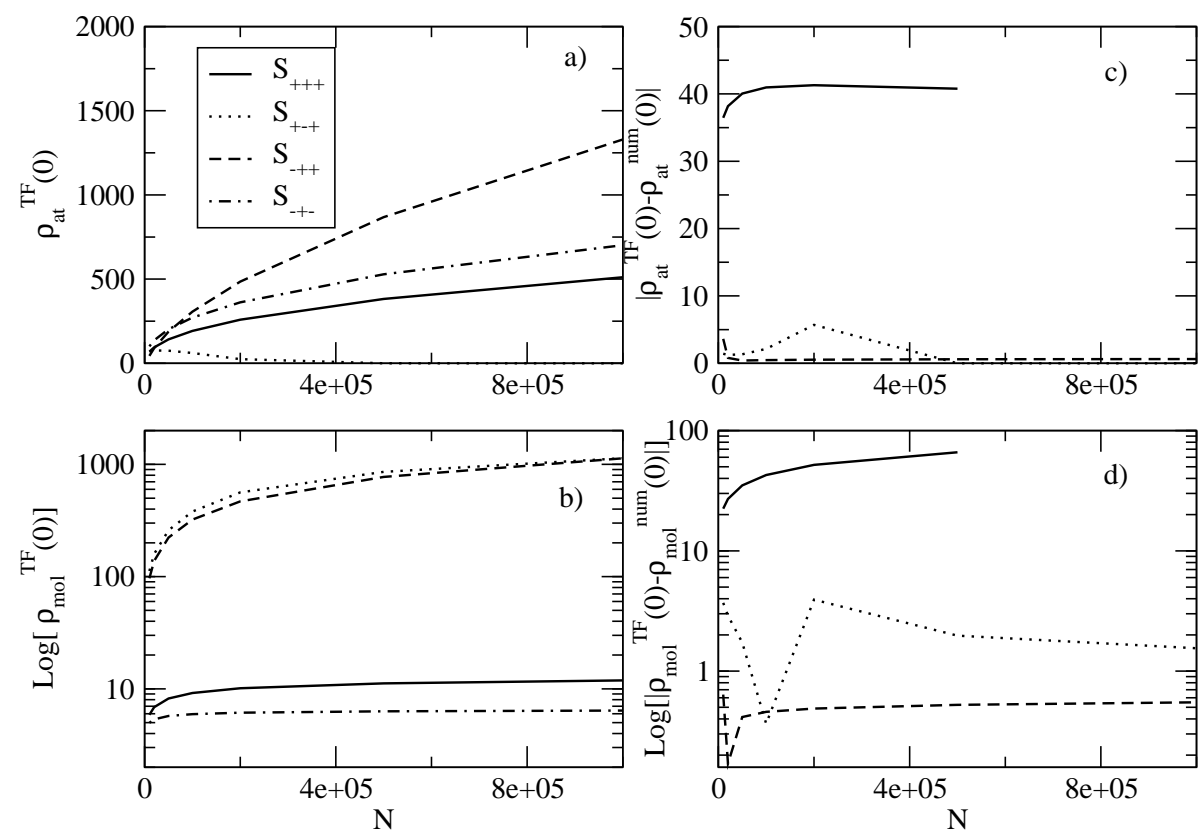

FIG. 2: The atomic (fig. a)) and molecular (fig.b)) central density in the TFA and the modular variation of atomic (fig. c)) and molecular (fig. d)) central densities, $\left|\rho_{\text {num }}^{a t}(0)-\rho_{T F}^{a t}(0)\right|$ and $\left|\rho_{\text {num }}^{\text {mol }}(0)-\rho_{T F}^{\text {mol }}(0)\right|$ as functions of the number of particles in four cases, $S_{+++}$(solid line), $S_{+-+}$(dotted line), $S_{-++}$(dashed line) and $S_{-+-}$(dotted-dashed line). There is no variation in the $S_{-+-}$because we do not have numerical solution for this case. We have not found an available numerical solution for $S_{+++}$at $N>7 \times 10^{5}$.

Likewise the $S_{+-+}$, the $S_{-++}$case in the TFA (see in the fig. 5) has good agreement to the corresponding numerical solution. Although there are first-derivative discontinuities at two distinct points for the TFA, namely at $r_{+}=\sqrt{2\left(\mu-\mu_{+}\right)}$and at $r_{1}=\sqrt{2 \mu}$. For $r<r_{+}$, the solution is mixed (atomic-molecular); for $r_{+}<r<r_{1}$, it is purely molecular. For $r>r_{1}$, there is a vacuum. Analogously to the $S_{+-+}$case with $\mu>\mu_{-}$, the numerical solution for the $S_{-++}$case is made up of an atom-molecule mixture. In the region of purely molecular density at the TFA, the atomic density provided by the numerical solution is almost null.

For the case $S_{-+-}$, (see in Fig. 6) there are discontinuities in the first derivatives of the densities in the TFA, as seen in the other solutions. Differently from the other cases, there is no available numerical solution when we used the TFA as a trial function for the parameters in the references [10, 13]. 

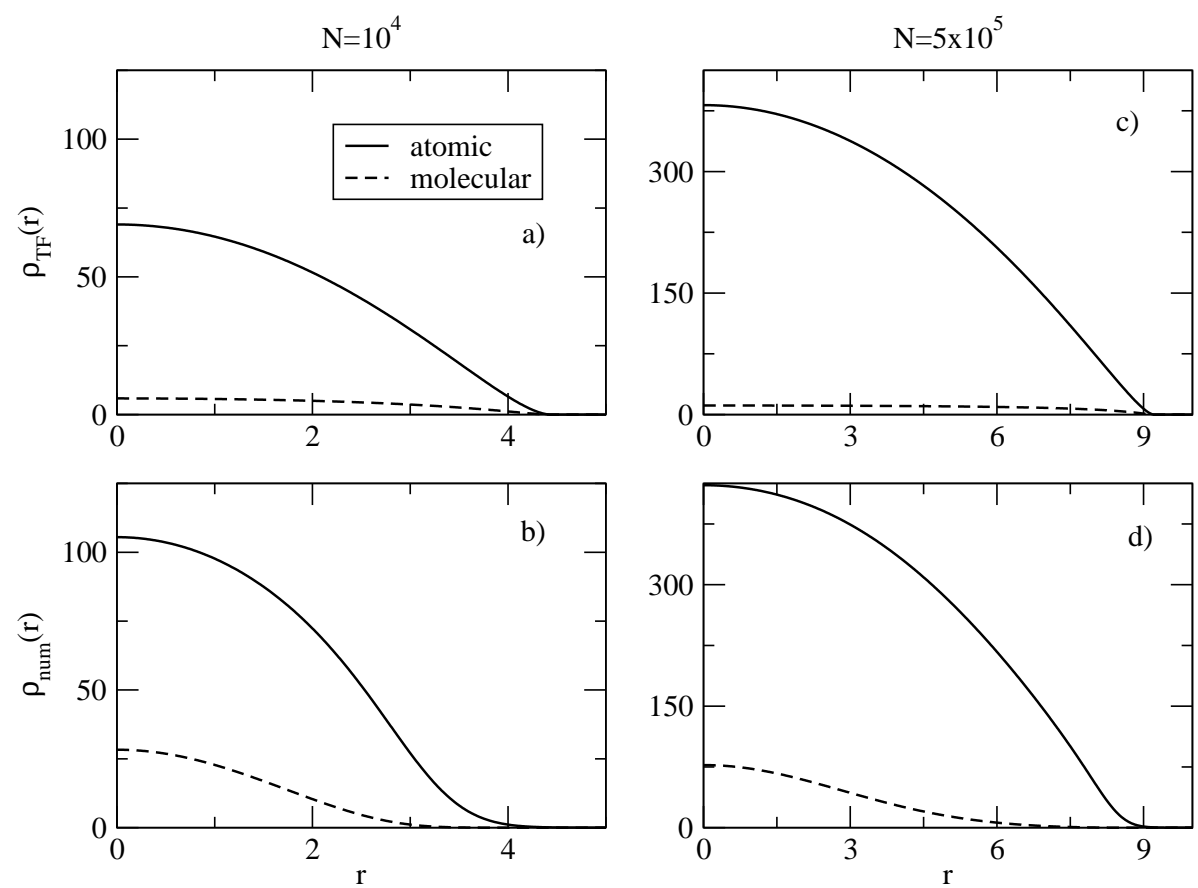

FIG. 3: Atomic density plot (solid line) and molecular density plot (dashed line) of the TFA and the numerical solution for $S_{+++}$considering $10^{4}$ particles (graphs a) and b)) and $5 \times 10^{5}$ particles (graphs c) and d)). We have chosen the initial parameters $U_{1}=0.1, U_{2}=0.036$ and $\alpha=1.08$.

\section{CONCLUSION}

We have considered a Bose gas in the AMBEC. The system consisting of atoms coherently coupled with their homonuclear diatomic molecules at zero temperature. For the TFA, we have analysed four possible spatial distributions in the conditions where the interspecies interactions are either negative or positive and where the coupling mode can be either in-phase or out-phase. For each solution at the TFA, we have used as trial function to obtain the numerical solution for the GPE.

As seen in the reference [13], the TFA spatial distribution was that it admits discontinuities in the derivatives of the densities in every case and presents discontinuities in the densities themselves in the $S_{+-+}$case. There are phase separations in every spatial distributions: a mixture-purely molecular interface in the cases $S_{+-+}$with $\mu=\mu_{-}$and $S_{-++}$, a vacuum-mixture interface in every case except for $S_{-++}$, where there is a vacuum-purely molecular separation.

The TFA is suitable with the numerical solution for the $S_{+-+}$and $S_{-++}$cases that present the phase separation. On the contrary of the solution at the TFA, the numerical solutions did not become zero neither for the molecular nor the atomic densities. Consequently, there was no 

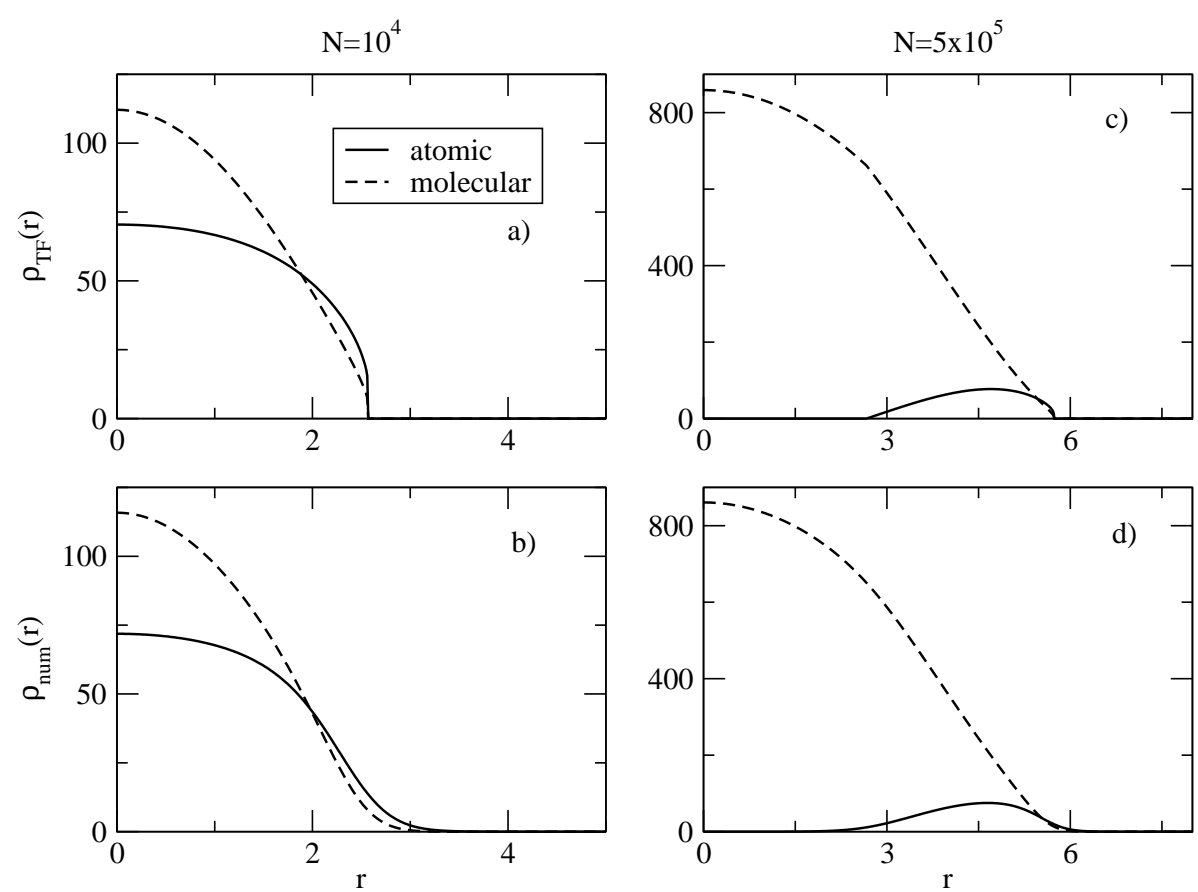

FIG. 4: Atomic density plot (solid line) and molecular density plot (dashed line) for $S_{+-+}$considering $10^{4}$ particles (graphs a) and b)) and $5 \times 10^{5}$ particles (graphs c) and d)). We have chosen the initial parameters $U_{1}=0.1, U_{2}=0.036$ and $\alpha=1.08$.

phase separations of any kind. Despite this difference, in the regions where the TFA has the purely molecular distribution, the numerical atomic density contributed with a low fraction of the total density compared to the molecular density. And, in the case where there is vacuum in the TFA, the numerical solution presents a low total density.

We have found the numerical solution corresponding to the $S_{+++}$case for $\mu<47$. For these numerical solutions, the atomic and molecular density profiles present a considerable difference between the TFA profiles. We have noted the atomic and molecular concentration in the center of the trap provided by the numerical solution is higher than the concentration generated by the TFA solution. This fact is unusual because we hope that the TFA solution will converge to the numerical solution when the number of particle increases. Likewise the cases above, the numerical solution has low total density in the region where the TFA presents vacuum.

In the condition of $\gamma=-1$ and $\beta=+1$ we have found two available solution in the TFA, $S_{-+-}$ and $S_{-++}$. For the $S_{-+-}$case, there is not numerical solution. As well as, the reference [10] have found only one numerical solution for this specific external condition. Thus we have related the $a m_{+}$state determining by the reference [10] with the $S_{-++}$case. 

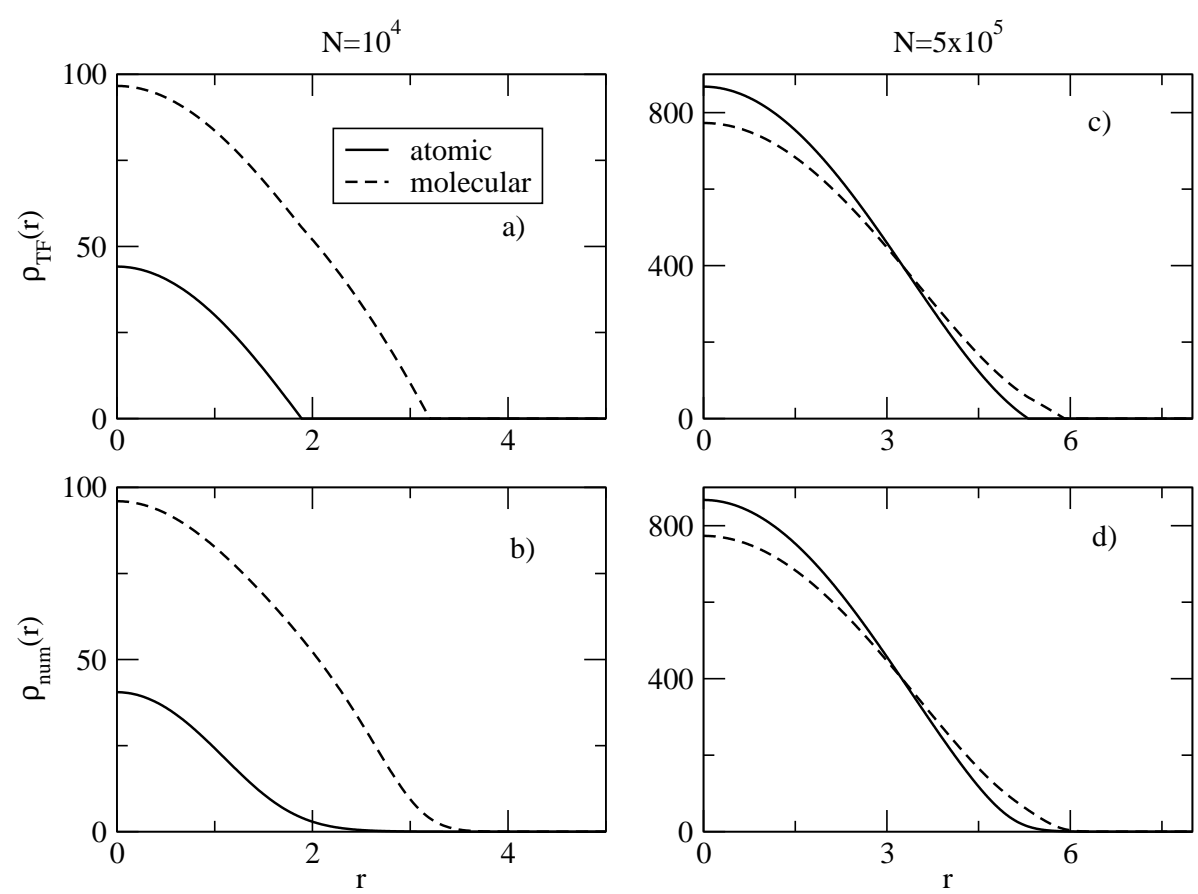

FIG. 5: Atomic density plot (solid line) and molecular density plot (dashed line) for $S_{-++}$considering $10^{4}$ particles (graphs a) and b)) and $5 \times 10^{5}$ particles (graphs c) and d)). We have chosen the initial parameters $U_{1}=0.062, U_{2}=0.012$ and $\alpha=1.09$.

We have noticed for the same kind of atom-molecule interaction $(\gamma)$, the solution with the less chemical potential have accordance between the TFA and the numerical solutions. For $\gamma=+1$, the atomic and molecular densities in the TFA and the numerical solution are agreed to each other only for $S_{+-+}$, while it does not happen in the $S_{+++}$cases. In the attractive atom-molecule interaction, $\gamma=-1$, the TFA solution of the $S_{-++}$case has accorded to the numerical solution. The $S_{-+-}$ not even have found the numerical solution.

For the relative phase equal to $\pi(\beta=-1)$ and the atom molecule interaction being attractive $(\gamma=-1)$, we could not obtain solutions for the TFA because $A M_{---}$and $A M_{--+}$both violated the physical and mathematical criteria for the acceptability of a solution. This situation corresponds to $a m_{-}^{t}$ state of the reference [10] have concluded that it cannot be determined by the TFA.

We believe that our work clarifies the importance of being judicious in the usage of the TFA. For the $S_{+-+}$and $S_{-++}$cases, the TFA is suitable to describe the system. However, though we have found the numerical solution only for $S_{+++}$case, the TFA can not describe the $S_{+++}$and $S_{-+-}$cases. 

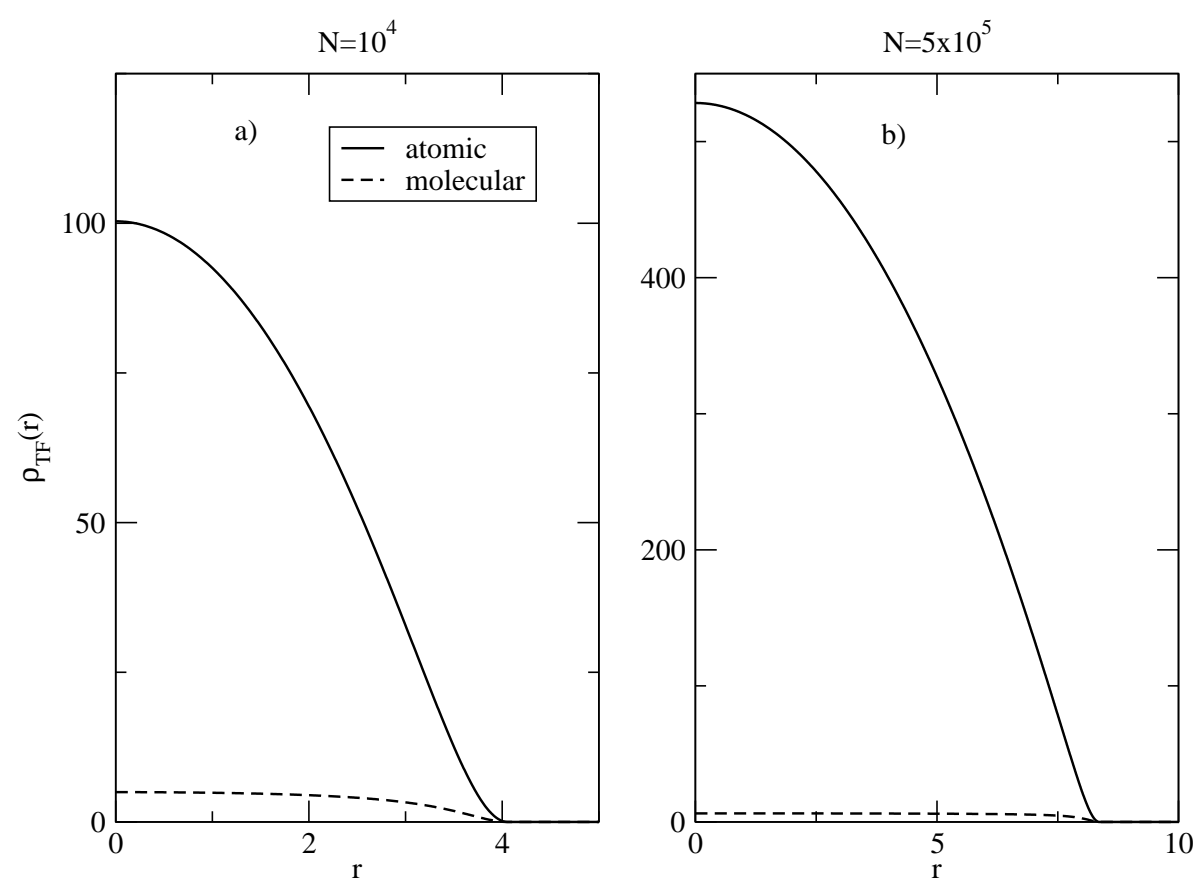

FIG. 6: Atomic density plot (solid) and molecular density plot (dashed) for $S_{-+-}$considering $10^{4}$ particles (graphs a)) and $5 \times 10^{5}$ particles (graphs b)) in the TFA. We have chosen the initial parameters $U_{1}=0.1$, $U_{2}=0.036$ and $\alpha=1.08$. There is no numerical solution for this case.

\section{Acknowledgments}

The authors would like to thank A. Gammal for tips on computing and A. F. R. de Toledo Piza for helping in the early phase of the present work. L. S. F. S. thanks FAPESP for financial support.

[1] M. H. Anderson et al., Science 269, 198 (1995).

[2] K. B. Davis, M. O. Mewes, M. R. Andrews, N. J. van Druten, D. S. Durfee, D. M. Kurn, and W. Ketterle, Phys. Rev. Lett. 75, 3969 (1995).

[3] C. C. Bradley, C. A. Sackett, J. J. Tollett, and R. G. Hulet, Phys. Rev. Lett. 75, 1687 (1995).

[4] R. Wynar, R. S. Freeland, D. J. Han, C. Ryu, and D. J. Heinzen, Science 287, 1016 (2000).

[5] C. J. Pethick, and H. Smith, Bose-Einstein Condensation in Dilute Gases (Cambrigde University Press, Cambrigde, 2002).

[6] M. Greiner, C. A. Regal and D. S. Jin, Nature 426, 537 (2003).

[7] C. Weber, G. Barontini, J. Catani, G. Thalhammer, M. Inguscio, and F. Minardi, Phys. Rev. A 78, 061601(R) (2008). 
[8] L. H. Lu and Y. Q. Lee, Phys. Rev. A 76, 053608 (2007).

[9] D. J. Heinzen et al., Phys. Rev. Lett. 84, 5029 (2000).

[10] B. J. Cusack, T. J. Alexander, E. A. Ostrovskaya, and Y. S. Kivshar, Phys. Rev. A 65, 013609 (2001).

[11] M. R. Matthews, D. S. Hall, D. S. Jin, J. R. Ensher, C. E. Wieman, E. A. Cornell, F. Dalfovo, C. Minniti, and S. Stringari, Phys. Rev. Lett. 81, 243 (1998).

[12] T. Isoshima, K. Machida, and T. Ohmi, Phys. Rev. A 60, 4857 (1999).

[13] X.-Q. Xu, L.-H. Lu, and Y.-Q. Li, Phys. Rev. A 79, 043604 (2009).

[14] R. Shibato, T. Nishimura, T. Watanabe, and T. Suzuki, Phys. Rev. A 84, 043627 (2011).

[15] P. A. Ruprecht, M. J. Holland, K. Burnett, and M. Edwards, Phys. Rev. A 51, 4704 (1995).

[16] S. K. Adhikari and P. Muruganandam, J. Phys. B 35, 2831 (2002).

[17] F. Dalfovo and S. Stringari, Phys. Rev. A 53, 2831 (1996).

[18] M. Brtka, A. Gammal, and L. Tomio, Phys. Lett. A 359, 339 (2006). 